\title{
Probing the helical edge states of a topological insulator by Cooper-pair injection
}

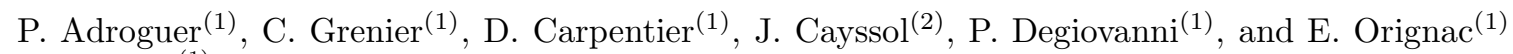 \\ ${ }^{(1)}$ Laboratoire de Physique, CNRS UMR5672, Ecole Normale Superieure de Lyon, \\ 46 Allée d'Italie, F-69364 Lyon Cedex 0\%, France and \\ (2) CPMOH, CNRS UMR 5798, Université Bordeaux-I, \\ 351, Cours de la Libération, F-33045 Talence, France
}

\begin{abstract}
We consider the proximity effect between a singlet s-wave superconductor and the edge of a Quantum Spin Hall (QSH) topological insulator. We establish that Andreev reflection at a QSH edge state/superconductor interface is perfect while nonlocal Andreev processes through the superconductor are totally suppressed. We compute the corresponding conductance and noise.
\end{abstract}

The prediction [1] and the observation [2, 3] of the Quantum Spin Hall (QSH) state in mercury telluride $(\mathrm{HgTe} / \mathrm{CdTe})$ heterostructures have triggered a great deal of excitation in the condensed matter community [4[6] since the QSH state realizes a two dimensional (2D) topologically ordered phase in the absence of magnetic field. The QSH state is distinguished from ordinary band insulators by the presence of a one-dimensional metal along its edge [7]. Owing to the dominant role of the spin-orbit interaction, this edge state provides a unique strictly one-dimensional metal where the spin is tied to the direction of motion of the carriers [8]. This so-called helical property and the associated time-reversal symmetry imply the absence of backscattering on non magnetic impurities.

So far the existence of the helical liquid has been confirmed by multiterminal transport measurements performed with normal leads [2, 3]. Since the QSH state exists under zero magnetic field, in contrast to the integer and fractional quantum Hall states, it can also be probed by the powerful methods of superconducting proximity effect [9]. Along these lines, Andreev spectroscopy has been recently suggested to characterize the quasi-relativistic dynamics of $2 \mathrm{D}$ bulk carriers in doped $\mathrm{HgTe} / \mathrm{CdTe}$ quantum wells [10]. Furthermore helical liquids might also be useful to analyze the entanglement of electrons injected from a singlet $s$-wave superconductor [11, 12].

In this Rapid Communication, we theoretically investigate the edge transport of a Quantum Spin Hall insulator in presence of a single superconducting probe. As a result of helicity conservation and absence of backscattering channel, we find that an electron can be either Andreev reflected as a hole, or transmitted as an electron. In a standard metal or in a carbon nanotube, there would be two additional possibilities whereby the electron can be reflected as an electron, or transmitted as a hole 13 19]. We compute the conductance and the noise associated to this partitioning in two outgoing channels, instead of four channels in standard 1D metals. The related experiments could be implemented readily using a side superconductor contacted to current $\mathrm{HgTe} / \mathrm{CdTe}$

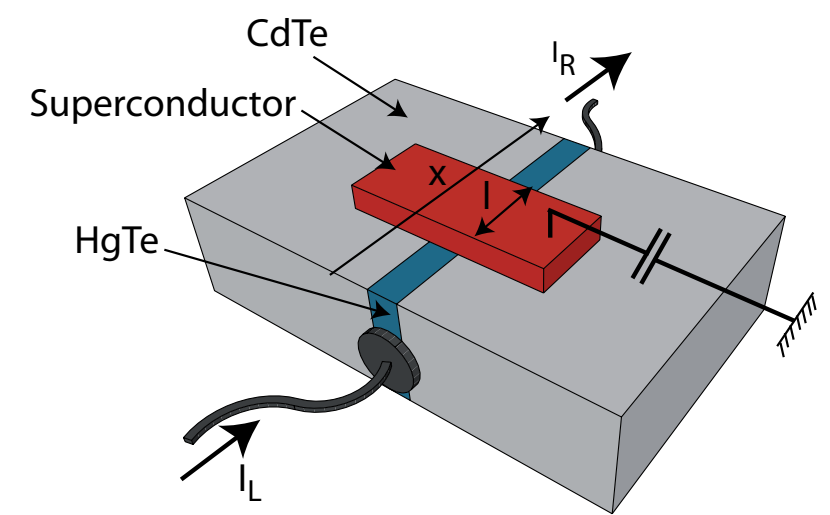

FIG. 1: (Color online). Schematic representation of the proposed experimental setup. The Quantum Spin Hall phase is realized in an inverted and insulating $\mathrm{HgTe} / \mathrm{CdTe}$ quantum well. Transport along the one-dimensional edge of the QSH phase is measured by a standard two terminals setup with normal electrodes. Between these two electrodes, a superconducting electrode is deposited over a length $l$ on one side of the sample.

samples [2, 3]. Our results also apply to other possible experimental realizations including the recently proposed inverted type II semiconductor quantum wells [20] and ultrathin $\mathrm{Bi}_{2} \mathrm{Se}_{3}$ films [21, 22]. Finally we contrast our results with Andreev transport through neutral Majorana fermions as realized at a triple interface between a ferromagnet, a superconductor and a topological insulator $[23$ 28].

In our proposed setup, a superconducting probe is deposited near an inverted $\mathrm{HgTe} / \mathrm{CdTe}$ quantum well thereby inducing superconducting correlations within the QSH edge state (Fig. 1). The counterpropagating electrons or holes are detected by distant normal metallic contacts. We assume a wide enough $\mathrm{HgTe}$ well so that scattering between opposite edges is absent [29]. The opposite limit of strong inter-edge scattering has been addressed in Refs. [11, 12]. In the absence of superconductivity, the single pair of gapless edge states is described 
by the one-dimensional Dirac Hamiltonian

$$
H_{0}=-i \hbar v_{F} \int_{-\infty}^{\infty} d x\left(\psi_{\uparrow}^{\dagger} \partial_{x} \psi_{\uparrow}-\psi_{\downarrow}^{\dagger} \partial_{x} \psi_{\downarrow}\right)
$$

where $h=2 \pi \hbar$ is Planck's constant and $v_{F}$ the Fermi velocity. Without any loss of generality, we have chosen the convention that the (pseudo)spin-up electrons associated with field operator $\psi_{\uparrow}(x)$ are right moving while the spin-down electrons are left moving. In contrast to a usual metal, there are no right movers with down spin or left movers with up spins. As a result, in a QSH edge, the product of the spin by the velocity is always positive which is called helicity conservation. These left and right movers are only well defined inside the bulk gap of the insulator which is typically $E_{g} \sim 1-30 \mathrm{meV}$ in $\mathrm{HgTe} / \mathrm{CdTe}$ quantum wells [2, 3].

We further assume that the superconductor induces a gap $\Delta(x)$ over a finite length $l$ of the helical liquid $[23-$ 28]. The edge transport is then described by the effective Hamiltonian

$$
H=H_{0}+\int_{0}^{l} d x\left(\Delta^{*}(x) \psi_{\downarrow}(x) \psi_{\uparrow}(x)+\text { H.c. }\right),
$$

where the amplitude of the proximity induced gap depends upon the coupling between the edge and the superconductor [30]. The induced gap amplitude $|\Delta|$ may reach at best the intrinsic gap of the superconductor, namely $|\Delta| \sim 0.1-1 \mathrm{meV}$ when using aluminium or niobium.

We first discuss qualitatively the available scattering processes in the opposite limits of long $(l \gg \xi=$ $\left.\hbar v_{F} /|\Delta|\right)$ and short $(l \lesssim \xi \sim 10-100 \mathrm{~nm})$ superconductor using only helicity conservation and time-reversal symmetry.

An electron with energy $\varepsilon<|\Delta|$ cannot be transmitted through a long superconductor $(l \gg \xi)$ since the penetration depth of the evanescent Bogoliubov quasiparticle in the superconductor is set by its coherence length $\xi$. Hence in a standard metal, this incident electron can be either reflected as an electron (electron backscattering) or as a hole (Andreev reflection) at a single normalsuperconducting (NS) metal interface [31]. Electron reflection changes the direction of propagation while conserving the spin: it is thus forbidden by helicity conservation in a QSH edge state. Due to unitarity, this absence of electronic backscattering implies Andreev reflection with unit probability even in presence of disorder and/or potential barrier at the interface. Such a perfect Andreev reflection is very difficult to achieve in standard metals where any defect or material parameters mismatch will induce a sizeable electron backscattering [32].

Interestingly, another kind of perfect Andreev reflection has been predicted recently for a Fermi lead coupled to a Majorana fermion at its end [27]. This perfect Andreev reflection results from the self conjugate property

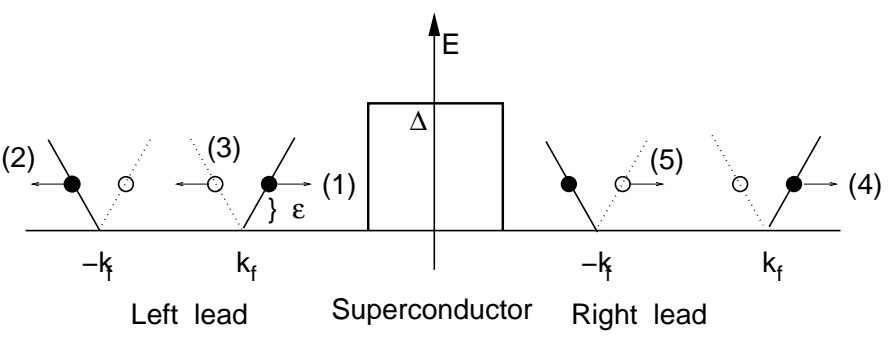

FIG. 2: (Color online). Scattering processes. With Fermi liquid leads, an incident electron (1) can be backscattered as an electron (2), reflected as a hole (local Andreev reflection) (3), transmitted as an electron (4) or transmitted as a hole (non local Andreev process) (5). In the QSH edge state, helicity conservation prevents electronic backscattering (2) and hole transmission (5). Furthermore, at low energy and for a wide superconductor, electron transmission (4) vanishes and only Andreev reflection (3) remains.

of the Majorana fermion which couples the electron and hole modes with equal amplitude. Nevertheless this resonant Andreev reflection requires a matching between the energy of the incident electron and the energy of the Majorana mode. By contrast, in our setup, the perfect Andreev reflection is achieved for all energies below the superconducting gap and relies only on the helical property of the lead.

Moreover, state-of-the art nanolithography allows for the realization of narrower superconducting regions $(l \lesssim$ $\xi)$ covering a metal strip [13, 14 or even a single carbon nanotube or nanowire [15, 16]. In such a normalsuperconducting-normal (NSN) geometry, subgap quasiparticles can also be transmitted. In a Fermi liquid lead, an incoming electron can be either transmitted as an electron (elastic cotunneling) or as a hole (non local Andreev process) [17 19]. The non local Andreev process have been evidenced recently in several experiments [15, 16]. Within the QSH edge, such Andreev transmission is again strictly forbidden by helicity conservation. Interestingly, in the presence of Majorana fermions, the Andreev transmission is restored and dominates the normal tunneling [24, 27].

Therefore along a QSH edge state, an incident electron can be only reflected as a hole by a superconducting barrier, or transmitted as an electron through it (Fig. 2). Using the Landauer-Büttiker formalism, we now provide the quantitative theory of this partitioning between Andreev reflection and normal transmission which holds for arbitrary superconductor length $l$, and energy $\epsilon$. We define the incoming fields, $\psi_{\uparrow, i}=\psi_{\uparrow}(x=0, t)$ and $\psi_{\downarrow, i}^{\dagger}=\psi_{\downarrow}^{\dagger}(x=l, t)$, in terms of the fermionic operators $\psi_{\uparrow, \downarrow}(x, t)=\psi_{\uparrow, \downarrow}\left(0, t \mp x / v_{F}\right)$ which capture the ballistic helical propagation within the edge state. Since only normal transmission and Andreev reflection are allowed the outgoing fields are defined as $\psi_{\uparrow, o}=\psi_{\uparrow}(x=l, t)$ and $\psi_{\downarrow, o}^{\dagger}=\psi_{\downarrow}^{\dagger}(x=0, t)$. The quasiparticle energy $\epsilon$ be- 


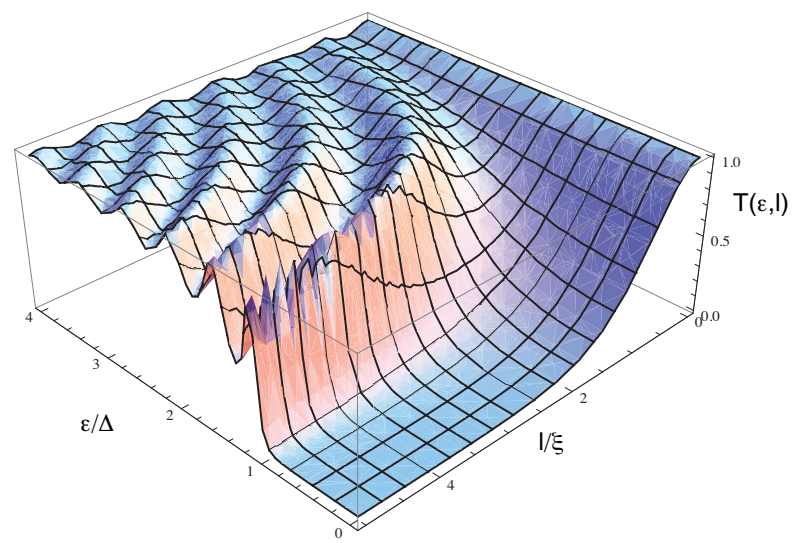

FIG. 3: (Color online). Plot of $T_{\epsilon}$ for a rectangular gap function $\Delta(x)=\Delta \theta(x) \theta(l-x)$ : the horizontal axis display $l / \xi$ and $\epsilon /|\Delta|$. For $l \gtrsim \xi$, the transmission vanishes for subgap electrons $\epsilon \leq|\Delta|$. Oscillations of the transmission coefficient for $\epsilon \geq|\Delta|$ can be interpreted as Fabry-Perot resonances of the Bogoliubov excitations through the superconducting barrier.

ing conserved, it is convenient to introduce the following Fourier representation:

$$
\psi_{\sigma}(x, t)=\int_{-\infty}^{\infty} \frac{d \epsilon}{2 \pi \hbar} e^{-i \epsilon t / \hbar} \psi_{\sigma}(x, \epsilon)
$$

Considering the solutions of the Dirac equation outside the barrier, and applying time reversal symmetry, we obtain:

$$
\begin{gathered}
\psi_{\uparrow, o}(\epsilon)=t(\epsilon) \psi_{\uparrow, i}(\epsilon)-\frac{r^{*}(\epsilon) t(\epsilon)}{t^{*}(\epsilon)} \psi_{\downarrow, i}^{\dagger}(-\epsilon), \\
\psi_{\downarrow, o}^{\dagger}(-\epsilon)=r(\epsilon) \psi_{\uparrow, i}(\epsilon)+t(\epsilon) \psi_{\downarrow, i}^{\dagger}(-\epsilon) .
\end{gathered}
$$

The scattering coefficients $r(\epsilon), t(\epsilon)$ which relate the incoming chiral fermionic fields to the outgoing ones must be obtained from the full solution of the one-dimensional Dirac equation associated with Eqs.(12). The probability for an electron of energy $\epsilon$ (with respect to the superconductor chemical potential) to be transmitted through the superconducting barrier is $T_{\epsilon}=|t(\epsilon)|^{2}$ and the probability for an electron of spin $\sigma$ and energy $\epsilon$ to be reflected as a hole of the same energy on the $-\sigma$ spin branch is $R_{\epsilon}=|r(\epsilon)|^{2}$. Current conservation always imposes $R_{\epsilon}+T_{\epsilon}=1$ irrespective of the specific shape of the pairing potential $\Delta(x)$.

Moreover in long superconducting segments, $l \gtrsim \xi$, electrons satisfying $\epsilon \leq|\Delta|$ experience total local Andreev reflection $\left(R_{\epsilon}=1\right)$ at each interface whereas for shorter superconducting regions, a finite electronic transmission is possible. On the other hand and for any length $l$, electrons of very high energy $\epsilon \gg|\Delta|$ are perfectly transmitted $\left(T_{\epsilon}=1\right)$ as pure electron-like quasiparticles through the superconducting barrier. For intermediate energies $\epsilon \gtrsim|\Delta|$, Bogoliubov quasiparticles experience Fabry-Perot like transmission resonances at discrete energies $\bar{\epsilon}_{n}$ given by condition $r\left(\bar{\epsilon}_{n}\right)=0$. In the case of a rectangular barrier $\Delta(x)=\Delta \theta(x) \theta(l-x)$, those resonances are located at $\hbar \bar{\epsilon}_{n}=\sqrt{\Delta^{2}+\left(\hbar v_{F} n \pi / l\right)^{2}}$ with $n$ integer (Fig. 3).

We now compute the conductance and noise of the three terminal setup when the left and right normal leads are biased at the respective potentials $V_{L}, V_{R}$ while the superconductor is grounded (Fig. 11). The condition that the electrons incoming from the reservoir are in thermal equilibrium is expressed as: $\left\langle\psi_{\sigma, i}^{\dagger}(\epsilon) \psi_{\sigma, i}\left(\epsilon^{\prime}\right)\right\rangle=\frac{2 \pi \hbar}{v_{F}} \delta(\epsilon-$ $\left.\epsilon^{\prime}\right) n_{\sigma}(\epsilon)$ and $\left\langle\psi_{\sigma, i}(\epsilon) \psi_{\sigma, i}^{\dagger}\left(\epsilon^{\prime}\right)\right\rangle=\frac{2 \pi \hbar}{v_{F}} \delta\left(\epsilon-\epsilon^{\prime}\right)\left(1-n_{\sigma}(\epsilon)\right)$ where $n_{\uparrow / \downarrow}(\epsilon)$ is the Fermi-Dirac distribution function for the $\uparrow$ (resp. $\downarrow$ ) incoming electrons, with chemical potential $\mu_{L}=e V_{L}$ (resp. $\mu_{R}=e V_{R}$ ). Along the edge state, the current operator is defined as $I(x, t)=$ $-e v_{F}\left(\psi_{\uparrow}^{\dagger} \psi_{\uparrow}-\psi_{\downarrow}^{\dagger} \psi_{\downarrow}\right)$. The current injected from the superconductor is described by the operator $I_{S}(t)=I_{R}(t)-$ $I_{L}(t)$, where $I_{L}(t)=I(x=0, t)$ and $I_{R}(t)=I(x=l, t)$ are the currents flowing in the left and right normal leads respectively (Fig. 1). Using Eqs. (4), the average current injected by the superconductor is found to be:

$$
\left\langle I_{S}\right\rangle=\frac{2 e}{h} \int_{-\infty}^{\infty} R_{\epsilon}\left(n_{\uparrow}(\epsilon)+n_{\downarrow}(-\epsilon)-1\right) d \epsilon .
$$

When $\mu_{R}=\mu_{L}=e V$, Eq. (5) leads to a differential conductance given by: $\left(\partial\left\langle I_{S}\right\rangle / \partial V\right)_{V=0}=\left(4 e^{2} / h\right) \times R_{\epsilon=0}$ increasing with $l$ from zero (no coupling to the superconductor for $l=0)$ to $4 e^{2} / h$ in the $l \gg \xi$ limit. Therefore we predict that the conductance $\left(\partial\left\langle I_{S}\right\rangle / \partial V\right)(V, T)$ must saturate at $4 e^{2} / h$ for low voltage/temperature $\left(\max \left(e V, k_{B} T\right) \ll \Delta, k_{B}\right.$ being the Boltzmann constant) which is the expected value for two perfectly Andreev reflecting $\mathrm{N} / \mathrm{S}$ interfaces in parallel.

The noise power $S_{S}(\omega)$ of the current injected from the superconductor, i.e. the Fourier transform of the autocorrelator $\left\langle I_{S}(0) I_{S}(t)\right\rangle$, can be computed from the scattering formalism with the help of Wick's theorem. At zero frequency, we find:

$$
\begin{aligned}
S_{S}(0) & =\frac{8 e^{2}}{h} \int_{-\infty}^{\infty} R_{\epsilon}\left(n_{\uparrow}\left(1-n_{\uparrow}\right)+n_{\downarrow}\left(1-n_{\downarrow}\right)\right) d \epsilon \\
& +\frac{8 e^{2}}{h} \int_{-\infty}^{\infty} R_{\epsilon} T_{\epsilon}\left(n_{\uparrow}+n_{\downarrow}-1\right)^{2} d \epsilon,
\end{aligned}
$$

where the shorthand notations $n_{\uparrow}=n_{\uparrow}(\epsilon)$ and $n_{\downarrow}=$ $n_{\downarrow}(-\epsilon)$ are used. The first term in Eq. (6) originates from the equilibrium thermal noise of the reservoirs whereas the second term is the nonequilibrium contribution to the superconducting current noise coming from the Andreev reflection/normal transmission partitioning. In the vanishing voltage limit $(V \rightarrow 0)$ only the first line of Eq. (6) contributes to the noise, and the Johnson-Nyquist relation $S_{S}(0)=4 k_{B} T\left(\partial\left\langle I_{S}\right\rangle / \partial V\right)_{V=0}$ is satisfied.

At zero temperature, only partially transmitted electrons $\left(T_{\epsilon} \neq 0,1\right)$ will generate a finite noise. For a rectangular pair potential $\Delta(x)=\Delta \theta(x) \theta(l-x)$, the noise 


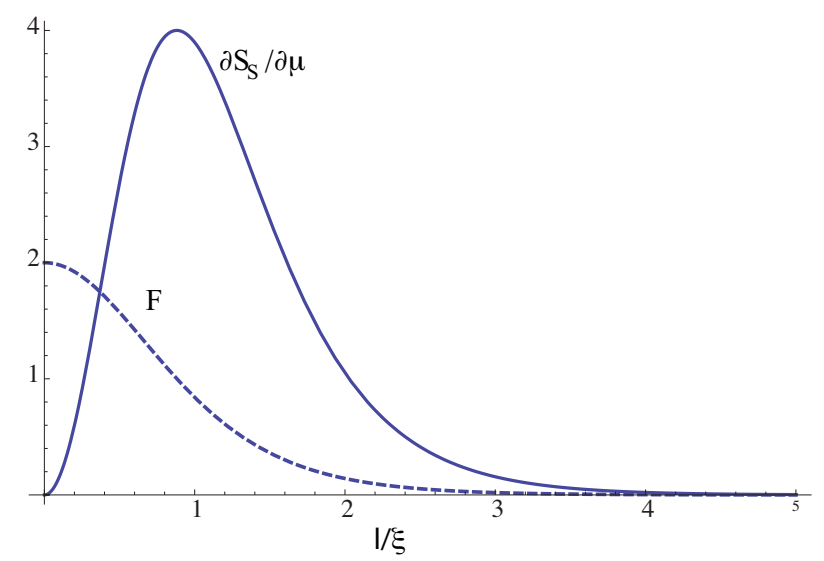

FIG. 4: (Color online). The noise response $\partial S_{S} / \partial \mu$ in units of $e^{2} / h$ (solid line) is maximal around $l \sim \xi$. The Fano factor $F=S_{S} / 2 e\left\langle I_{S}\right\rangle$ (dashed line) as a function of $l / \xi$.

response to a low bias $e V_{L}=e V_{R}=\mu$,

$$
\frac{\partial S_{S}}{\partial \mu}=\frac{16 e^{2}}{h} \frac{\sinh ^{2}(l / \xi)}{\cosh ^{4}(l / \xi)}
$$

reaches the maximal value of $4 e^{2} / h$ for a superconductor width corresponding to an equal partitioning between the local Andreev reflection and the normal transmission processes, namely $T_{\epsilon=0}=R_{\epsilon=0}=1 / 2$ (Fig. 4, solid line). At zero temperature and vanishing bias voltage $V \rightarrow 0$, the corresponding Fano factor $F=$ $S_{S} / 2 e\left\langle I_{S}\right\rangle=2 T_{\epsilon=0}$ is twice the transmission probability through the barrier (Fig. 4, dashed line), in agreement with the transfer of charges $2 e$ between the superconductor and the QSH edge. For long superconducting segments, $l \gtrsim \xi$, one obtains two uncorrelated and noiseless QSH/superconductor interfaces where for subgap electrons the quasiparticle currents are converted into supercurrent through perfect normal Andreev reflection.

In conclusion, we have considered a junction between a superconductor electrode and a QSH phase. The conservation of helicity and the resulting absence of backscattering manifest themselves into a perfect Andreev reflection for long junction in the subgap regime. This leads to unique signatures of the helical nature of the edge states, including in particular the existence of noiseless injected currents on both sides of long QSH/S/QSH junctions. Our model assumed that the QSH edge states were not reconstructed in the presence of the superconductor. In particular, we have neglected the possibility of the formation of new QSH edge channels or a local 2D metallic puddle beneath the superconductor. Such non-universal effects might prevent the observation of full Andreev reflection. However, their consideration in a selfconsistent Bogoliubov-de Gennes theory, which depends on the full bandstructures of both the superconductor and the topological insulator, is beyond the scope of this Rapid Communication.
Finally we further anticipate interesting two-particle interference effects in the high frequency regime. In particular a fermionic version of the Hong-Ou-Mandel experiment 33] could be implemented using single electron and single hole excitations obtained from Lorentzian voltage pulses applied to the left and right reservoirs [34].

J.C. thanks Bjoern Trauzettel, Patrik Recher and Marine Guigou for stimulating discussions. J.C. acknowledges funding from the Institut de Physique Fondamentale in Bordeaux. This work was supported by the Agence Nationale de la Recherche under Grant No. ANR-07-NANO-011: ELEC-EPR (J.C.) and under the program blanc ISOTOP (D.C., J.C. and E.O.).

[1] B. Bernevig, T. L. Hughes, and S.-C. Zhang, Science 314, 1757 (2006).

[2] M. Konig, S. Wiedmann, C. Brune, A. Roth, H. Buhmann, L. W. Molenkamp, X.-L. Qi, and S.-C. Zhang, Science 318, 766 (2007).

[3] A. Roth, C. Bruene, H. Buhmann, L. Molenkamp, J. Maciejko, X.-L. Qi, and S.-C. Zhang, Science 325, 294 (2009).

[4] X.-L. Qi and S.-C. Zhang, Physics Today 63, 33 (2010).

[5] J. E. Moore, Nature 464, 194 (2010).

[6] M. Z. Hasan and C. L. Kane (2010), arXiv:1002.3895.

[7] C. L. Kane and E. J. Mele, Phys. Rev. Lett. 95, 226801 (2005).

[8] C. Wu, B. Bernevig, and S.-C. Zhang, Phys. Rev. Lett. 96, 106401 (2006).

[9] M. Tinkham, Introduction to Superconductivity (McGraw-Hill New York, 1996).

[10] M. Guigou and J. Cayssol, arXiv:1005.1055.

[11] M.-S. Choi, arXiv:1002.3144.

[12] K. Sato, D. Loss, and Y. Tserkovnyak, arXiv:1003.4316.

[13] D. Beckmann, H. Weber, and H. Löhneysen, Phys. Rev. Lett. 93, 197003 (2004).

[14] P. Cadden-Zimansky and V. Chandrasekhar, Phys. Rev. Lett. 97, 237003 (2006).

[15] L. Hofstetter, S. Csonka, J. Nygard, and C. Schonenberger, Nature (London) 461, 960 (2009).

[16] L. G. Herrmann, F. Portier, P. Roche, A. L. Yeyati, T. Kontos, and C. Strunk, Phys. Rev. Lett. 104, 026801 (2010).

[17] J. Byers and M. Flatté, Phys. Rev. Lett. 74, 306 (1995).

[18] S. G. den Hartog, C. M. A. Kapteyn, B. J. van Wees, and T. Klapwijk, Phys. Rev. Lett. 77, 4954 (1996).

[19] G. Deutscher and D. Feinberg, Appl. Phys. Lett. 76, 487 (2000).

[20] C. Liu, T. Hughes, X.-L. Qi, K. Wang, and S.-C. Zhang, Phys. Rev. Lett. 100, 236601 (2008).

[21] C.-X. Liu, H. Zhang, B. Yan, X.-L. Qi, T. Frauenheim, X. Dai, Z. Fang, and S.-C. Zhang, Phys. Rev. B 81, 041307 (2010).

[22] H.-Z. Lu, W.-Y. Shan, W. Yao, Q. Niu, and S.-Q. Shen, Phys. Rev. B 81, 115407 (2010).

[23] L. Fu and C. L. Kane, Phys. Rev. Lett. 100, 096407 (2008).

[24] J. Nilsson, A. R. Akhmerov, and C. W. J. Beenakker, 
Phys. Rev. Lett. 101, 120403 (2008).

[25] A. R. Akhmerov, J. Nilsson, and C. W. J. Beenakker, Phys. Rev. Lett. 102, 216404 (2009).

[26] L. Fu and C. L. Kane, Phys. Rev. Lett. 102, 216403 (2009).

[27] K. T. Law, P. A. Lee, and T. K. Ng, Phys. Rev. Lett. 103, 237001 (2009).

[28] Y. Tanaka, T. Yokoyama, and N. Nagaosa, Phys. Rev. Lett. 103, 107002 (2009).

[29] B. Zhou, H. Lu, R. Chu, S. Shen, and Q. Niu, Phys. Rev. Lett. 101, 246807 (2008).
[30] T. Stanescu, J. Sau, R. Lutchyn, and S. D. Sarma, arXiv:1002.0842.

[31] A. Andreev, Sov. Phys. JETP 19, 1228 (1964).

[32] G. E. Blonder, M. Tinkham, and T. Klapwijk, Phys. Rev. B 25, 4515 (1982).

[33] Z. Y. Ou, C. K. Hong, and L. Mandel, Phys. Rev. A 36, 192 (1987).

[34] L. Levitov, H. Lee, and G. Lesovik, J. Math. Phys. 37, 4845 (1996). 